\title{
Ambulatory electrocardiographic monitoring in patients with transient focal cerebral ischaemia
}

\author{
DAVID A FRANCIS, ${ }^{*}$ JAMES R HERON, $\dagger$ MALCOLM CLARKE $\ddagger$ \\ From the Departments of Neurology, ${ }^{*}$ and Cardiology, $\ddagger$ North Staffordshire Hospital Centre, \\ Stoke-on-Trent, and the Department of Postgraduate Medicine, $\dagger$, University of Keele, Keele, Staffordshire, \\ UK.
}

SUMMARY Sixty-four patients with transient ischaemic attacks were investigated for the presence of an associated cardiac arrhythmia. 24-hour ambulatory monitoring showed arrhythmia in $41 \%$ of patients where the standard ECG had shown arrhythmia in only $25 \%$. Significant arrhythmias were found in $21 \%$ of patients with focal transient ischaemic attacks on prolonged monitoring as compared to $7 \%$ in a control group. Where arrhythmias were specifically treated there was abolition or improvement of neurological symptoms.

Much attention has been directed recently to the association of cardiac arrhythmias with diffuse cerebral ischaemia and symptoms of syncope, confusion or convulsive seizures. ${ }^{1-3}$ The frequency and significance of focal transient ischaemic attacks due to cardiac arrhythmia has however been less fully studied. ${ }^{4}$ This study sets out to determine the incidence and relevance of significant arrhythmias in patients who present with focal transient ischaemic attacks.

\section{Patients and Methods}

Sixty-four patients with recurrent transient ischaemic attacks were classified according to the site of cerebral ischaemia. A transient ischaemic attack was defined as an acute focal neurological symptom lasting less than 24 hours and thought to be due to vascular disease of an arterial embolic or thrombotic kind. Group 1 were patients who had experienced transient ischaemic attacks in the internal carotid artery territory; the symptoms included amaurosis fugax, dysphasia, and transient unilateral motor or sensory disturbances. Group 2 was of patients with transient ischaemic attacks in the vertebrobasilar territory, with binocular visual symptoms, diplopia, dysarthria, bilateral motor or sensory disturbances and ataxia. Group 3 was of patients with mixed symptoms of the types reported by patients in Groups 1 and 2.

All patients were admitted and the following investiga-

Address for reprint requests: Dr JR Heron, Department of Neurology, North Staffordshire Hospital Centre, Stoke-on-Trent. ST4 7LN, UK.

Received 7 June 1983 and in revised form 24 September 1983 Accepted 3 October 1983 tions performed; standard haematology, biochemistry, measurement of plasma viscosity and fasting serum lipids, radiography of skull, chest and cervical spine and a CT brain scan. Carotid angiography was performed where appropriate.

Each patient had a resting 12-lead electrocardiogram (ECG) and an ambulatory 24-hour electrocardiographic record. Records were made on magnetic cassette tape using an Oxford Medilog cassette recorder with the electrodes applied to give an ECG configuration similar to $V_{s}$. The tapes were analysed with a Pathfinder arrhythmia detector and any observed abnormality was printed out on standard ECG paper for formal "blind" analysis by a cardiologist (MC).

A control group of 72 ambulatory in-patients matched for approximate age were also studied. Forty-nine controls had primary neurological disorders not known to be related to cardiac or cerebrovascular disease, for example myelopathy, radiculopathy and peripheral nerve entrapment; 23 had non-neurological diagnoses such as patients convalescing from limb fractures or gastrointestinal disorders. A resting ECG and 24-hour ECG record were obtained for each patient and analysed as above.

Patients were assessed for the presence of cardiovascular disease such as a past history of coronary insufficiency, angina, myocardial infarction and hypertension; smoking habit was also documented.

Standard ECGs were classified as showing (1) ischaemic changes, (2) arrhythmia or (3) normal record (table 1). We accepted arrhythmia on 24-hour records as: significant, potentially significant, or not significant, using the same criteria as Jonas et al..$^{5}$ Ventricular and atrial ectopic beats were considered relevant if the tapes showed one or more epochs with a frequency that was greater than one ectopic beat in five sinus beats over a period of 60 seconds (fig 1) or when they occurred in couplets or salvoes ${ }^{5}$ (complicated extrasystoles, see table 2 ). 

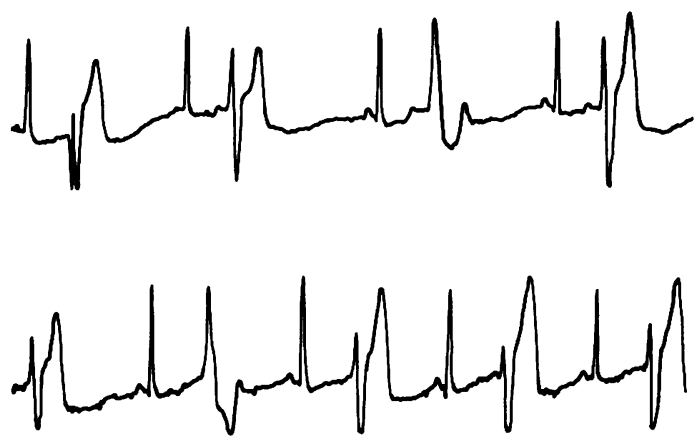

Fig 1 24-hour tape showing frequent multifocal ventricular ectopic beats (standard ECG: sinus rhythm)

Table 1 12-lead ECG analysis. Arrhythmias seen on 24-hour ECG tapes

\begin{tabular}{lcclcc}
\hline Standard ECG & $\begin{array}{c}\text { None Not } \\
\text { significant }\end{array}$ & $\begin{array}{l}\text { Potentially } \\
\text { significant }\end{array}$ & Significant & Total \\
\hline Ischaemic & 13 & 1 & 0 & 2 & 16 \\
Arrhythmia & $3 \dagger$ & 6 & 1 & 5 & 15 \\
Normal & 24 & 4 & 1 & 4 & 33 \\
Total & $40 \dagger$ & 11 & 2 & 11 & 64 \\
\hline
\end{tabular}

† Standard ECG revealed coronary sinus rhythm-significant arrhythmia- where 24-hour tape was normal.

$\mathrm{VE}=$ ventricular ectopic

Table 2 Arrhythmias detected by ambulatory 24-hour ECG monitoring

\begin{tabular}{lll}
\hline & Patient & Control \\
\hline Significant: & 3 & - \\
Tachybradycardia syndrome & 3 & 2 \\
Paroxysmal supraventricular tachycardia & 3 & - \\
Sinus bradycardia ( $\$$ 40 bpm) & 0 & - \\
Paroxysmal ventricular tachycardia & 2 & 1 \\
Complicated ventricular ectopics (see text) & 3 & 2 \\
Potentially significant: & 2 & 1 \\
Uncomplicated ventricular ectopics (> 5 bpm) & 2 & 1 \\
Not significant: & 2 & 4 \\
Sinus arrhythmia & 5 & 16 \\
Sinus bradycardia (> 40 bpm) & & \\
Infrequent VEs/SVEs & $40 \dagger$ & 49 \\
Normal: & 64 & 72 \\
Sinus rhythm (<2 VEs) &
\end{tabular}

† Standard ECG revealed coronary sinus rhythm-significant arrhythmia-where 24-hour tape was normal.

$\mathrm{VE}=$ ventricular ectopic

\section{Results}

The 64 transient ischaemic attack patients comprised 34 males and 30 females. There were 20 patients in Group 1, 27 in Group 2 and 17 in Group 3 . The mean age of the males was 61.5 years (age range 45-76) and of the females 67.4 years (age range 32-72). The control group comprised 42 males, mean age 59.6 years (age range $37-88$ ) and 30 females, mean age 65.9 years (age range $40-80$ ).

Cardiac arrhythmias were present in $41 \%$ of all patients on prolonged monitoring in comparison to $32 \%$ in the control population (table 2). Haemodynamically significant arrhythmias occurred in $20.8 \%$ of these patients as compared to $6.9 \%$ of our control group. The incidence of all arrhythmias was not different between males and females nor clinical groups, although "significant" arrhythmias were twice as common in patients experiencing carotid transient ischaemic attacks as compared to vertebrobasilar attacks (30\% and $15 \%$ respectively). Significant arrhythmias showed an equal proportion of supraventricular and ventricular rhythm disturbances with a peak incidence in the 7 th and 8th decades. Potentially significant and nonsignificant arrhythmias were mainly ventricular in origin.

Standard ECGs showed an arrhythmia in 15 patients $(23 \%)$. Two showed atrial fibrillation, the remainder showed either isolated ectopic beats or sinus bradycardia. Sixteen patients had ischaemic changes only, eight of whom had conduction defects, and there were 33 normal records (table 1). Of the 15 patients who had shown arrhythmia in the standard ECG, prolonged monitoring indicated significant arrhythmia in six. In those patients with ischaemic changes only, two patients, both with conduction defects, were subsequently shown to have significant arrhythmias. In the 33 patients with a normal ECG record nine were shown to have an arrhythmia on prolonged monitoring, of which five were significant arrhythmias. The standard ECG had no predictive value in the determination of a significant arrhythmia on the 24-hour record.

The study as a whole showed an equal number of patients who had a preceding history of cardiovascular disease and patients with no such history. The distribution of all arrhythmias was approximately equal in these two groups (54\% and $46 \%$ respectively), this was also true for significant arrhythmias which did not show an association with preceding heart disease (Chi square test $\chi_{2}^{2}=0.39, \mathrm{p}>0.75$ ).

Of 37 patients (Groups 1 and 3) who had carotid transient ischaemic attacks, 17 had confirmatory evidence of carotid stenosis and 10 had significant arrhythmias. Although six of these 10 patients with arrhythmias had demonstrable carotid stenosis this association did not reach statistical significance (Fisher Exact probability test $P=0 \cdot 17$ ).

Of 44 patients (Groups 2 and 3 ) with vertebrobasilar transient ischaemic attacks there were $66 \%$ with radiological evidence of severe or moderate cervical spondylosis. Eight patients had significant arrhythmias on prolonged monitoring, six of whom had advanced spondylosis but again the association was not statistically significant $(P=0 \cdot 28)$. 


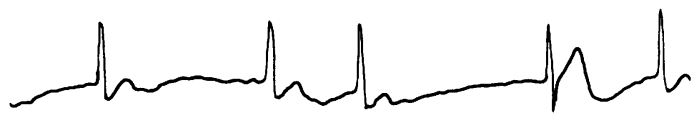

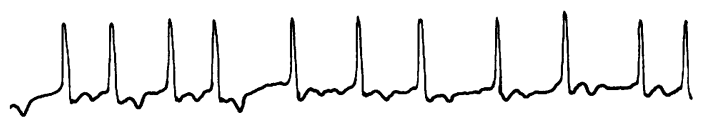

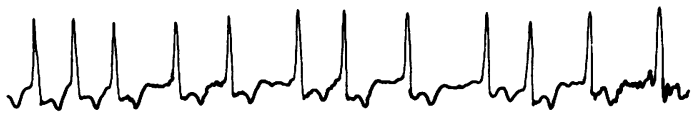

Fig 2 24-hour tape showing atrial fibrillation with tachybradycardia syndrome in a 76-year-old man presented with carotid transient ischaemic attacks (standard ECG: atrial fibrillation).

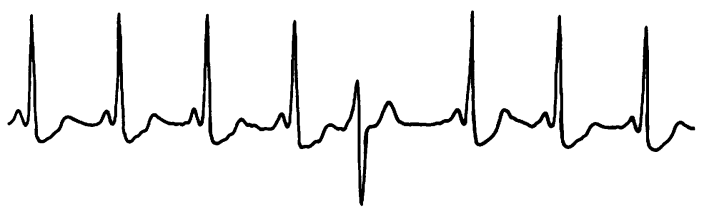

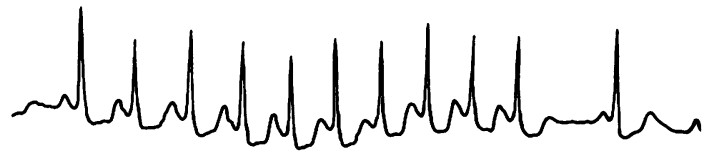

Fig 3 Isolated episode of paroxysmal supraventricular tachycardia in a 70-year-old man who presented with transient episodes of right hemisensory loss. (standard ECG: right bundle branch block, left axis deviation).

Plasma hyperviscosity and hyperlipidaemia were not associated with an increased incidence of arrhythmia $\left(\chi_{2}^{2}=1.29 \mathrm{p}>0.5\right.$ and 1.95 , $0.25<\mathrm{p}<0.5$ respectively). Cigarette smokers showed a sixfold increase in the presence of significant arrhythmias as compared to non-smokers, $85.7 \%$ as to $14.3 \% \quad \chi_{2}^{2}=5 \cdot 42$, $0.05<p<0.1$ (a larger sample with the same proportions would give a significant result at the $5 \%$ level).

Of 12 patients with significant arrhythmias, four had endocardial pacemakers inserted (tachybradycardia syndrome-three patients, fig 2; paroxysmal supraventricular tachycardia-one patient, fig 3) and remain asymptomatic after 18 months.

Three patients were treated medically, one is symptom-free on disopyramide (fig 4) after 24 months), the second patient had rapid atrial fibrillation and has experienced fewer transient ischaemic attacks following digitalisation (follow up 24
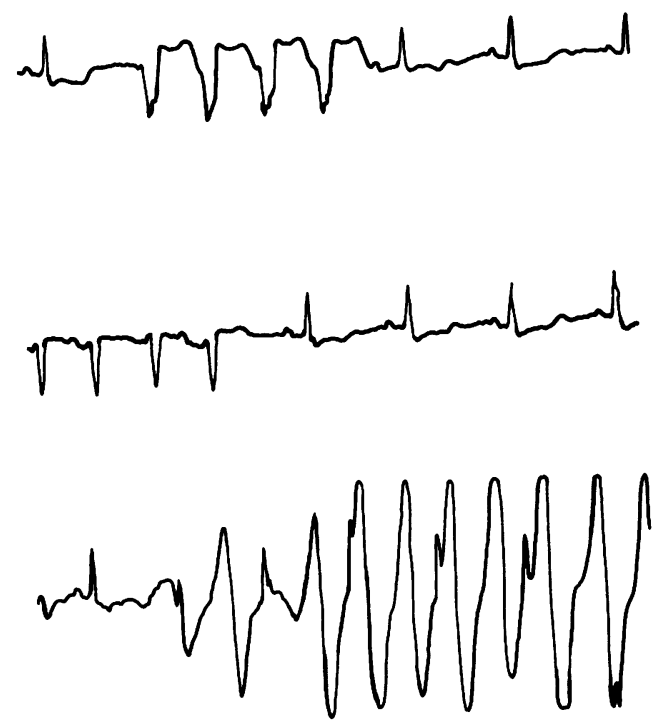

Fig 4 Runs of ventricular tachycardia in a 57-year-old woman experiencing frequent episodes of transient hemiplegia. (standard ECG: sinus rhythm). Symptoms abolished following disopyramide therapy.

months). The third patient in this group died from a mesenteric embolus two months after starting disopyramide treatment for paroxysmal ventricular tachycardia. Before treatment she had experienced at least one disabling carotid transient ischaemic attack per month for the preceding six months. During the short period between starting treatment and her sudden death she had been symptom free. Of the remaining patients, one became asymptomatic following carotid endarterectomy despite the persistence of his rhythm disturbance (coronary sinus rhythm), two patients improved spontaneously without treatment (complicated ventricular ectopics) and two patients defaulted from follow-up. The two patients with "potentially significant" arrhythmias were treated with aspirin and dipyridamole. Both reported an improvement of symptoms despite continuing rhythm disturbances and remain under review.

\section{Discussion}

Transient ischaemic attacks often occur as the result of microemboli from extracranial sources, such as the heart or the major arteries in the neck. Less frequently haemodynamic changes in cardiac output are responsible and these may occur as a result of cardiac arrhythmia, which can be intermittent. Atrial and ventricular arrhythmias are accepted as causing attacks of syncope and seizures; ${ }^{56}$ the evi- 
dence relating focal transient ischaemic attacks to disturbances of cardiac rhythm is however less secure. McHenry et $a l^{2}$ and McAllen and Marshall ${ }^{3}$ reported a significant proportion of patients with transient focal cerebral ischaemia and abnormalities of cardiac rhythm, whereas Reed et al ${ }^{4}$ stressed the rarity of focal neurological symptoms in patients with symptomatic arrhythmias. Recent reports have noted that cardiac rhythm abnormalities may be common and asymptomatic in elderly people ${ }^{7}$ which suggests that cardiac arrhythmias play only a subsidary role in the aetiology of transient ischaemic attacks in this group.

In our study significant arrhythmias were present in $21 \%$ of patients with focal transient ischaemic attacks on prolonged monitoring as compared to $7 \%$ of the control group. When considering all arrhythmias they occurred in $41 \%$ of such patients and $32 \%$ of our control population.

Although an increased incidence of arrhythmia has been reported in association with pre-existing cardiac disease, ${ }^{89}$ our results show an equal frequency in patients with and in those without heart disease. Also, in contrast to some reports ${ }^{30}$ the standard ECG in our study was not reliable in predicting a significant rhythm disturbance on prolonged monitoring (Chi square test). In accordance with the findings of Luxon et al, 9 nine of our patients with normal standard ECGs had unsuspected arrhythmias demonstrated upon monitoring and in over half of these cases the arrhythmia was haemodynamically significant.

Cardiac arrhythmia may cause a sudden reduction in blood flow across a stenosed extracranial or intracranial artery and produce focal cerebral ischaemia. ${ }^{4}$ It is therefore necessary to evaluate the role of rhythm disturbances when considering individual patients and defined clinical groups of patients with cerebrovascular disease. In our "carotid" group $60 \%$ of significant arrhythmias occurred in patients with internal carotid artery stenosis. In the "vertebrobasilar" group $66 \%$ of the significant arrhythmias occurred in patients who had moderate or severe cervical spondylosis. We suggest that in the presence of a reduced cardiac output from arrhythmia, other factors, such as co-existent carotid stenosis, may precipitate the induction of focal cerebral ischaemic events. In support of this hypothesis, it may be relevant that one of our patients with carotid stenosis became asymptomatic after endarterectomy despite persistence of his arrhythmia and that a second patient, with bilateral carotid stenosis, became asymptomatic after treatment with disopyramide and the cessation of her paroxysmal tachycardia (fig 4).

No patient experienced a transient ischaemic attack during monitoring even when the period of recording was extended to 48 hours. There is good evidence to indicate however that arrhythmias of short duration occurring in the absence of associated neurological symptoms, may herald the later development of arrhythmia-induced clinical attacks. ${ }^{5}$ We have found an increased incidence of these brief "heralding" arrhythmias in patients experiencing focal transient ischaemic attacks as compared to a control population.

In this study, no association was found between plasma hyperviscosity or hyperlipidaemia and the presence of a symptomatic cardiac arrhythmia. By contrast regular cigarette smoking, a treatable risk factor, showed six times the incidence of important rhythm disturbances in our patients as compared to non-smokers.

Our results indicate that the presence of short duration arrhythmias on ambulatory ECG monitoring justifies anti-arrhythmic treatment and consideration of pacemaker implantation in selected patients with cerebrovascular disease.

We thank Miss K Heraty, Senior ECG technician and her staff for their co-operation and technical assistance.

\section{References}

1 Van Durme JP. Tachyarrhythmias and transient cerebral ischaemic attacks. Am Heart $J$ 1975;89:538-40.

${ }^{2}$ McHenry LC, Toole JF, Miller HS. Long-term ECG monitoring in patients with cerebrovascular insufficiency. Stroke, 1976;7:264-9.

${ }^{3}$ McAllen PM, Marshall J. Cardiac dysrhythmia and transient cerebral ischaemic attacks. Lancet, 1973;1:1212-4.

4 Reed RL, Sickert RG, Meridith J.Rarity of transient focal cerebral ischaemia in cardiac dysrhythmia. JAMA 1973;223:893-5.

5 Jonas S, Klein I, Dimant J. Importance of Holter monitoring in patients with periodic cerebral symptoms. Ann Neurol 1977;1:470-4.

- Schott GD, McLeod AA, Jewitt DE. Cardiac arrhythmias that masquerade as epilepsy. $\mathrm{Br}$ Med $\mathrm{J}$ 1977;1:1454-7.

${ }^{7}$ Rai GS. Cardiac arrhythmias in the Elderly. Age Ageing. 1982;11:113-5.

${ }^{8}$ Hinkle LE, Carver ST, Stevens M. The frequency of asymptomatic disturbances of cardiac rhythm and conduction in middle-aged men. Am J Cardiol 1969;24:629-50.

' Luxon LM, Crowther A, Harrison MJG, Coltart DJ. Controlled study of 24-hour ambulatory electrocardiographic monitoring in patients with transient neurological symptoms. $J$ Neurol Neurosurg Psychiatry 1980;43:37-41.

10 Walter PF, Reid SD, Kass Wenger N. Transient cerebral ischaemia due to arrhythmia. Ann Intern Med 1970;72:471-4. 